\title{
$\beta R$ IS A TOPOLOGICAL GALOIS SPACE
}

\author{
P. L. SHARMA
}

\begin{abstract}
The result given in the title of this note is proved. An example of two nonhomeomorphic Hausdorff Galois spaces having isomorphic homeomorphism groups follows.
\end{abstract}

Fletcher and Snider [3] introduced the class of topological Galois spaces and conjectured that any two Hausdorff spaces of this class would be homeomorphic if the full homeomorphism groups of the spaces were isomorphic. Later, Fletcher and McCoy [2] doubted the validity of this conjecture and suggested some special subclasses of the above-mentioned class, where the conjecture might possibly hold.

In this note we prove that $\beta R$ is a topological Galois space and that consequently $R$ and $\beta R$ are two nonhomeomorphic Galois spaces having isomorphic full homeomorphism groups. Note that the space $\beta R$ is not representable in the sense of [2] and thus the validity of this conjecture in the class of representable Galois spaces remains undetermined.

$H(X)$ will denote the group of all homeomorphisms of a topological space $X$. Recall that a topological space $X$ is called a topological Galois space, provided, given any $x \in X$ and closed $F$ not containing $x$, there exists $h \in H(X)$ such that $h(y)=y$ for each $y \in F$ and $h(x) \neq x$.

$R$ denotes the real line with its usual topology and $\beta R$ is its Stone-Čech compactification. The underlying set of $\beta R$ is the set of closed ultrafilters on $R$, and we will not distinguish between a fixed closed ultrafilter and the corresponding point of $R$, so that we let $R \subset \beta R$. Since the only points of $\beta R$ having a countable neighborhood base are the points of $R, H(R)$ and $H(\beta R)$ can be easily seen to be isomorphic [4].

THEOREM 1. $\beta R$ is a Hausdorff Galois space.

Proof. Let $\mathscr{F}_{0}$ be a closed ultrafilter on $R$ and let $Z_{0}$ be any closed subset of $R$ such that $Z_{0} \notin \mathscr{F}_{0}$. Then $\left\{\mathscr{F} \in \beta R: Z_{0} \in \mathscr{F}\right\}$ is an arbitrary basic closed subset of $\beta R$ not containing the point $\mathscr{F}_{0}$ of $\beta R$. Let

$$
A=\{x \in R: 2 n \leqslant x \leqslant 2 n+1 \text { for some integer } n\},
$$

and

Received by the editors January 29, 1976 and, in revised form, March 11, 1976.

AMS (MOS) subject classifications (1970). Primary 54H15.

Key words and phrases. Topological Galois space, homeomorphism group. 


$$
B=\{x \in R: 2 n-1 \leqslant x \leqslant 2 n \text { for some integer } n\} .
$$

Then $A \cup B=R \in \mathscr{F}_{0}$ and so at least one of $A$ and $B$ is in $\mathscr{F}_{0}$. Without loss of generality, we suppose $A \in \mathscr{F}_{0}$. As $Z_{0} \notin \mathscr{F}_{0}$ we can find $F_{0} \in \mathscr{F}_{0}$ such that $F_{0} \cap Z_{0}=\varnothing$. Then $F=F_{0} \cap A \in \mathscr{F}_{0}$ and $F \cap Z_{0}=\varnothing$. We can find an open set $G$ of $R$ containing $F$ such that (i) $\bar{G} \cap Z_{0}=\varnothing$, and (ii) $G$ contains no interval of length greater than 2 . This is clearly possible because the closed set $F$ is contained in $A$ and is disjoint from $Z_{0}$. Express $G$ as a union of countable number of pairwise disjoint open intervals $I_{n}=\left(a_{n}, b_{n}\right), n=1,2$, $3, \ldots$ As $a_{n}, b_{n} \notin F$ and $F$ is closed in $R$, we can find a closed interval $\left[c_{n}, d_{n}\right] \subset\left(a_{n}, b_{n}\right)$ such that $F \cap\left(a_{n}, b_{n}\right) \subset\left[c_{n}, d_{n}\right]$. Now define a function $g: R \rightarrow R$ as follows:

$$
g(x)= \begin{cases}x & \text { if } x \notin G, \\ \frac{c_{n}-a_{n}}{d_{n}-a_{n}}\left(x-a_{n}\right)+a_{n} & \text { if } a_{n}<x<d_{n}, \\ \frac{c_{n}-b_{n}}{d_{n}-b_{n}}\left(x-b_{n}\right)+b_{n} & \text { if } d_{n} \leqslant x<b_{n} .\end{cases}
$$

Clearly $g$ is a one-to-one mapping from $R$ onto $R$; and as $g$ and $g^{-1}$ are monotonically increasing, $g \in H(R)$. Also $g(E)=E$ for each $E$ $\subset Z_{0}$ and $g(F) \cap F=\varnothing$. Let $g^{*}$ be the Stone extension of $g$ to $\beta R$. Then $g^{*} \in H(\beta R), g^{*}(\mathscr{F})=\mathscr{F}$ for each $\mathscr{F}$ containing $Z_{0}$ and $g^{*}\left(\mathscr{F}_{0}\right) \neq \mathscr{F}_{0}$. The proof is now complete.

Notation. Let $X$ be a Tychonoff space. Then $\gamma X=\beta X-X$, where $\beta X$ is the Stone-Čech compactification of $X$. The subspace of $R$ consisting of all nonnegative real numbers is denoted by $R^{+}$.

THEOREM 2. $\gamma R$ and $\gamma R^{+}$are Hausdorff Galois spaces.

Proof. Let $h \in H(\beta R)$. Then $\left.h\right|_{\gamma R} \in H(\gamma R)$; and it clearly follows that $\gamma R$ and each of its connected components are Hausdorff Galois spaces. It is well known [for example, see Gillman and Jerison, Rings of continuous functions, p. 92] that the disconnected space $\gamma R$ is the union of two disjoint continua each homeomorphic to $\gamma R^{+}$. It follows that $\gamma R^{+}$is a Hausdorff Galois space. The following corollary should be compared to Theorem 2 of [1].

Corollary. Let $X=[0,1)$; then $\gamma X$ is a Hausdorff Galois space.

\section{REFERENCES}

1. David P. Bellamy and Leonard R. Rubin, Indecomposable continua in Stone-Čech compactifications, Proc. Amer. Math. Soc. 39 (1973), 427-432.

2. P. Fletcher and R. A. McCoy, Galois spaces, representable spaces and strongly locally homogeneous spaces, Fund. Math. 73 (1971/72), 85-91. MR 45 \#2650.

3. P. Fletcher and R. L. Snider, Topological Galois spaces, Fund. Math. 68 (1970), 143-148. MR 42 \# 1089.

4. J. de Groot, Groups represented by homeomorphism groups. I, Math. Ann. 138 (1959), 80-102. MR 22 \#9959. 